\title{
EL FUTURO DE LAS HUMANIDADES
}

\author{
Adela Cortina
}

Universidad de Valencia, España

\section{LAS DOS CULTURAS: CIENTÍFICOS RESPONSABLES, INTELECTUALES LUDITAS}

En el año 1959, Charles Percy Snow, físico y novelista británico, pronuncia su célebre conferencia sobre "Las dos culturas y la revolución científica", que produjo un gran revuelo. Snow, en su conferencia, entendía el término "cultura" en dos sentidos. El primero de ellos se refería al desarrollo intelectual, al cultivo del entendimiento, al sentido en que Coleridge hablaba de "cultivation" o de "armonioso desarrollo de aquellas cualidades humanas y facultades que caracterizan a nuestra humanidad" (Snow 1977b, 74).

Ciertamente, no andaba lejos esta acepción del término "cultura" de la que Ortega asignaba a la palabra "humanidad", a esa humanitas de la que dice ser probablemente un invento verbal de Cicerón, que significaría primero aproximadamente lo que en el siglo XIX se decía con "civilización" y "cultura": un cierto sistema de comportamientos humanos que se consideraban ejemplares y a los que los hombres grecolatinos de la época helenística creían "por fin" haber llegado (Ortega 1961, 1). Solo que a partir de este punto, Ortega trata de acompañar al término "humanitas" a lo largo de la Edad Media hasta llegar al siglo XX, mientras que Snow da por bueno que la "humanitas" incluye entre sus cualidades tanto las que se desarrollan en el estudio científico como las que desarrollan aquellos a los que suele llamarse "intelectuales".

Se trataría entonces de dos subculturas, que comparten el conjunto del bagaje humano, es decir, el ámbito de la curiosidad por el mundo natural y el aprecio por los sistemas simbólicos de pensamiento. Alcanzar la humanidad 
en plenitud exigiría cultivar estas cualidades, pero lamentablemente cada una de las subculturas dejaría languidecer una parte de ellas.

En una segunda acepción, que es la que interesará a Snow en mayor medida, el vocablo "cultura" se refiere a "todo grupo de seres humanos que vive en un mismo ambiente, y está vinculado por hábitos comunes, supuestos comunes y común manera de vivir" (Snow 1977b, 75). Desde esta perspectiva, en el mundo del saber topamos con dos grupos culturales, los intelectuales y los científicos, que al parecer desarrollan formas de vida diferentes. Y no deja de ser curioso que Snow señale como una de las características de la forma de vida científica el interés por la investigación, mientras que el ejemplo que menciona a cuento de los intelectuales es que no permiten que se les censure una palabra. Lo cual es cierto en este segundo caso, pero no lo es que a los intelectuales no les interese la investigación. Un aspecto del mayor interés para el tema que nos ocupa: el futuro de lo que se ha dado en llamar "humanidades".

Y continuando con Snow, tres problemas se plantearán en la convivencia de estos dos grupos, a su juicio: 1) Los intelectuales tratan de monopolizar toda la cultura y la identifican con la cultura tradicional, que es la suya. 2) Por otra parte, y ésta es la crítica más dura que Snow lanza a los intelectuales, son luditas por antonomasia o, lo que es idéntico, irresponsables. Por eso no han entendido la revolución industrial ni la han aceptado, cuando es, a su juicio, la única esperanza de mejora que existe para los pobres. Mientras los científicos trabajan con optimismo por un futuro mejor, porque les preocupa el bien de los hombres, los intelectuales se envuelven en la capa de su pesimismo y demonizan esa revolución que es la que en realidad puede mejorar la situación de los menos aventajados. 3) Por último, entre las dos culturas existe una gran incomunicación. Es de primera necesidad que las dos culturas entren en diálogo, y la educación es, obviamente, un buen medio para lograrlo.

La conferencia de Snow provocó toda suerte de críticas y también de adhesiones, y el autor se sintió invitado a retomar el tema cuatro años más tarde en "Las dos culturas: un segundo enfoque". De este trabajo solo quisiéramos mencionar que el autor alude a la emergencia de una tercera cultura, que aglutina a intelectuales, interesados por el modo en que viven los seres humanos desde un grupo de saberes, que son la historia social, la sociología, la demografía, las ciencias políticas, la economía, el gobierno, la psicología, la medicina y desde artes sociales, como la arquitectura. A estos intelectuales les importan los efectos humanos de la revolución científica y están componiendo una nueva cultura (Snow 1977b, 81 y 82). 
Qué duda cabe de que esos saberes habían venido cobrando desde hacía tiempo un perfil propio y convirtiéndose en lo que ha dado en llamarse las "ciencias sociales", como han reconocido, entre otros, Jerome Kagan, psicólogo del desarrollo y emérito en Harvard, en su libro The Three Cultures: Natural Sciences, Social Sciences and the Humanities in the 21st. Century. Un libro en cuya portada consta expresamente "Revisiting C.P. Snow", porque el autor quiere tomar el pulso al tema medio siglo después de la controvertida conferencia de Snow.

\section{LAS TRES CULTURAS: CARACTERÍSTICAS Y RELACIONES MUTUAS}

Para cumplir su objetivo, el libro de Kagan se propone sobre todo caracterizar cada una de las culturas, esbozar las diferencias que existen entre ellas y también describir las relaciones que guardan entre sí en términos de valoración social. Le acompañaremos brevemente en estos tres propósitos, porque son centrales para esclarecer el futuro de las humanidades.

En lo que hace a la caracterización de las tres culturas y sus diferencias mutuas, Kagan propone nueve parámetros que le sirven de referencia (Kagan 2009, 2-5):

1) Cómo se plantea en ellas el grado en que la predicción, la explicación o la descripción de un fenómeno es el producto principal de la investigación. Las ciencias naturales se interesan por predecir, explicar o describir los fenómenos naturales; las ciencias sociales, por predecir y explicar las conductas humanas y los estados psicológicos; las humanidades, por comprender las reacciones humanas y los significados en función de la cultura, la época histórica y la historia vital.

2) Las fuentes de evidencia en que se basan las inferencias y el grado de control que se puede ejercer sobre las condiciones en que se capta la evidencia. Las ciencias naturales basan sus inferencias en observaciones de entidades materiales, experimentalmente controladas; las ciencias sociales, en las conductas, enunciados verbales y en medidas biológicas; y las humanidades se basan en textos y en conductas humanas, recogidos en condiciones de control mínimo.

3) El vocabulario empleado. En el caso de las ciencias naturales, conceptos semánticos y matemáticos, cuyos referentes son entidades materiales y que trascienden las situaciones particulares; las ciencias sociales se 
servirían de constructos referidos a los rasgos, estados psicológicos y conductas de individuos o grupos, y aceptarían las constricciones que impone el contexto a la hora de generalizar; mientras que las humanidades recurrirían a conceptos que se refieren a la conducta humana y los contextos impondrían serias restricciones a las inferencias.

4) El grado en que influyen en las cuestiones las condiciones sociales producidas por acontecimientos históricos. Que sería un grado mínimo, en el caso de las ciencias naturales; modesto, en el de las sociales, y serio, en el de las humanidades.

5) El grado en que los valores éticos impregnan las cuestiones y las conclusiones. Que, según Kagan, es mínimo en el caso de las ciencias naturales y más elevado en el de las otras dos culturas.

6) El grado en que dependen del apoyo financiero sea del gobierno, sea de la industria. Sería muy elevado en el primer caso, modesto en el segundo y relativamente independiente en el tercero.

7) La probabilidad de que el académico trabaje solo, con uno o dos académicos más, o como miembro de un equipo. En este punto los científicos naturales suelen trabajar en equipo, tanto pequeño como amplio, los científicos sociales trabajan en colaboración en menor medida y los humanistas suelen trabajar en solitario.

8) La contribución a la economía nacional que, en opinión de Kagan, es elevada en el primer caso, modesta en el segundo y mínima en el tercero.

9) Por último, también difieren las tres culturas en los criterios de los miembros del grupo para juzgar un cuerpo de trabajo como elegante o bello.

Naturalmente, la caracterización que hace Kagan de las tres culturas es discutible. Resulta difícil encuadrar en alguna de ellas las ciencias de la salud, desprecia la presencia de los valores éticos y la incidencia del contexto histórico en las ciencias naturales, y no reconoce que las culturas se entreveran entre sí. Pero, con todo, la caracterización es útil para comprender dos aspectos centrales del tema que ocupa a este trabajo: la dificultad de aplicar parámetros para medir la calidad de las investigaciones en humanidades, que queda muy acreditada por los tres primeros rasgos diferenciales, y la escasa valoración que se hace de sus aportaciones a la hora de distribuir los recursos económicos, por creer que no precisan apoyo financiero, que los humanistas siempre trabajan en solitario y, sobre todo, que los estudios de humanidades contribuyen muy poco al crecimiento de la economía nacional. 
Estas creencias están relacionadas con la valoración social que cada ámbito del saber merece, valoración que ha sufrido un cambio sustancial desde los tiempos de Snow. Las humanidades ya no ocupan el lugar más elevado, las ciencias de la salud son sumamente apreciadas, también los científicos sociales merecen en ocasiones un alto aprecio, sobre todo los economistas, juristas y psicólogos, las ciencias naturales ligadas a la aplicación tecnológica, por supuesto, y las básicas parecen tener más dignidad que precio. ¿A qué se debe ese descenso en el aprecio por las humanidades?

\section{¿DECLIVE DE LAS HUMANIDADES?}

Kagan ofrece cuatro razones (Kagan 2009, 226-228) para explicar el declive de las Humanidades, de las que sólo mencionaremos dos por su interés.

Entiende Kagan, y creo que lleva razón, que los humanistas han perdido su sentido de la profesionalidad cuando los postmodernos argumentan que cualquier persona puede filosofar, escribir una novela, una historia, una biografía, hacer aguda crítica filológica o interpretar acontecimientos históricos sin necesidad de haber adquirido conocimientos específicos de las humanidades. E incluso, creo yo, han ayudado a devaluarlas quienes han proclamado que la filosofía ha de disolverse en las ciencias sociales o en la literatura, con la irresponsabilidad del funcionario que sabe que, pase lo que pase con los saberes, él va a seguir manteniendo su puesto. Y un buen día, cuando los gobiernos deciden reducir los horarios de filosofía en la Enseñanza Media, los mismos que habían predicado la necesidad de su disolución se revuelven indignados contra tal medida y acusan al gobierno correspondiente de querer una ciudadanía inculta, sin capacidad crítica, para poder manipularla mejor.

¿Es que los saberes que componen las humanidades no cuentan con métodos específicos; con términos y conceptos peculiares que es preciso conocer para manejarse en ellos, componiendo un vocabulario propio que conviene enriquecer, pero al que no se puede renunciar; con tradiciones que ayudan a resolver mejor los problemas actuales; con un modo propio de comprobar la verdad, la adecuación o la validez de las propuestas?

Qué duda cabe de que todo ser humano tiene capacidad de reflexionar, de saber que sabe y tomar distancia frente a sus conocimientos, criticándolos. Pero tampoco cabe duda de que unas propuestas filosóficas dan mejor cuenta de la realidad que otras, y para tomar decisiones sobre ello hay que conocerlas y conocerlas bien. Como tampoco es dudoso que unas interpretaciones 
históricas son más plausibles que otras teniendo en cuenta el contexto, la información y la cultura de la época. La práctica de las humanidades requiere conocimientos específicos, rigurosos, aunque no exactos, como diría Ortega, requiere profesionalidad, en el mejor sentido del término.

Por otra parte, según Kagan, el descenso de las humanidades en la valoración social se debe también a que los científicos invaden el terreno de los humanistas. En primer lugar, los científicos sociales, pero cada vez más los neurocientíficos, según los cuales, las "verdaderas" medidas de la percepción, la memoria, el pensamiento y la emoción son los perfiles cerebrales, no los informes de conducta o verbales.

Y esto es, en cierta medida, verdad, pero no nuevo. A lo largo de la historia, las ciencias que han gozado de mayor éxito en su época han intentado dar razón de la vida toda, con un afán proselitista asombroso. En los últimos tiempos un buen número de neurocientíficos repite la hazaña de querer fundar una filosofía de la vida sobre bases cerebrales, de forma que el método de descripción, explicación y comprobación empírica pueda dar cuenta de la conducta humana en su conjunto. Pero si esto no es una novedad, sí lo es que el alcance de los medios de comunicación en una sociedad globalizada pone en manos de científicos y pseudocientíficos la posibilidad de propalar infundios, como el que consiste en decir que por fin la ciencia va a mostrar la verdad del mundo y las orientaciones legítimas para la acción, y todo ello - presuntamente- sobre bases empíricamente comprobables (Cortina 2011 y 2012).

Los científicos sensatos exponen su saber con grandes cautelas y únicamente apuntan que conocer mejor el funcionamiento de nuestro cerebro nos ayuda a saber más sobre nosotros mismos y, por lo tanto, a organizar mejor nuestras vidas. A fin de cuentas, las comprobaciones empíricas conducen a muy modestos resultados, que tienen que ser interpretados desde una comprensión del posible sentido que los seres humanos dan a los fenómenos observados, y desde luego no permiten transitar a lo que se debe hacer desde un punto de vista moral y político. Comprensión e interpretación del sentido son indispensables para conocer el mundo humano, como también lo es descubrir fundamentos racionales para la acción a la hora de bosquejar lo que debería ser. Éstas son tareas propias de las humanidades, en trabajo conjunto con los demás saberes.

Afortunadamente, la incomunicación entre las culturas, en la que Snow veía el principal problema, deja de ser una realidad poco a poco. La revolución de las éticas aplicadas en el campo del desarrollo, la economía y la empresa, 
las biotecnologías, la práctica médica y ese inmenso número de ámbitos al que llega la realidad de una reflexión ética, inserta ya en las instituciones sociales y políticas, da fe de que la comunicación existe (Cortina/García-Marzá 2003). Entre los humanistas sigue habiendo un buen número acomodado en el discurso de la crítica ludita, pero cada vez más crece el de los que, preocupados por la causa de los menos aventajados, incorporan cuanto pueda venir del progreso científico y se aprestan a colaborar en la tarea de ponerlo al servicio de la humanidad. No existe ya, y no debe existir, la incomunicación entre las culturas. Pero tampoco debe ninguna de ellas diluirse en las otras, porque cada una tiene su tarea específica y su lugar irreemplazable en el diálogo de los saberes por un mundo más justo.

En este orden de cosas, otro punto de los que sugiere Kagan merece una detenida discusión: su afirmación de que la contribución a la economía nacional es mínima en el caso de las humanidades. Una afirmación que es radicalmente falsa, y que invita de modo contundente a los diseñadores de políticas científicas a invertir poco en humanidades por la falsa creencia de que no son rentables. "Invertir en $\mathrm{I}+\mathrm{D}+\mathrm{i}$ " no parece ser una acción relacionada con las humanidades.

Unido esto a la convicción de que los humanistas apenas necesitan recursos, porque no precisan complicados instrumentos, sino que prácticamente les basta con algún ordenador, se entiende que es irrazonable invertir en humanidades. Cuestiones ambas que deben ser abordadas con seriedad. La primera, porque no es verdad que las humanidades no incidan en la economía nacional, y la segunda, porque no solo los humanistas necesitan medios, sino que la distribución de los recursos económicos sobre la base de "medidas de calidad" de la producción científica acaba siendo una cuestión también de reconocimiento. Sin embargo, aquí nos limitaremos a hablar de la fecundidad de las humanidades.

\section{LA FECUNDIDAD DE LAS HUMANIDADES}

En 1948, Ortega redacta un borrador para su proyectado Boletín del Instituto de Humanidades, en el que recuerda que si las disciplinas de humanidades quieren cobrar de nuevo su auténtico vigor es preciso integrar la ciencia en su unidad orgánica, procurando compensar de algún modo la dispersión especialista (Ortega 1962, 443). Tras el origen ciceroniano de la "humanitas", a que ya nos hemos referido, este sustantivo singular se convierte en plural, 
en esas humanidades que no son ya una forma de vida, sino un conjunto de conocimientos y enseñanzas, "en que la vida transparece sólo oblícuamente", porque la atención va dirigida sobre todo a las palabras: su tema son las obras poéticas, retóricas, históricas, jurídicas, didácticas de griegos y romanos, hasta que en el siglo XV la actividad intelectual está dominada por la gramática.

Con el tiempo se hace urgente estudiar la realidad humana en lo que tiene de específica, y entonces se acuñan las expresiones "moral sciences" o bien "morals" en el mundo anglosajón, "ciencias morales y políticas" en el francés e hispano, "Geisteswissenschaften" en el mundo germano, y Ortega propone denominar "humanidades" a estos saberes que se ocupan de hechos exclusivamente humanos. Frente al conocimiento naturalista, las humanidades no llevan a consecuencias directa y claramente útiles, sino que proporcionan un conocimiento estricto, pero no exacto; trabajan en hechos, pero tratan de articularlos desde el sentido, que es la materia inteligible en el mundo humano. ¿Qué pueden aportar tales conocimientos al progreso de las sociedades? A mi juicio, hacen aportaciones como las siguientes.

En primer lugar, tienen en cuenta esa intersubjetividad humana, sin la que no existen ni ciencia objetiva ni política legítima. Las humanidades en este nuestro siglo XXI (la filosofía, las filologías, la historia y la literatura) tienen por objeto reflexionar sobre esa intersubjetividad humana que es el vínculo entre sujetos cada uno con su peculiar identidad, sujetos que precisan reconocerse recíprocamente para hacer ciencia natural y social y para hacer conjuntamente la vida cotidiana. Como muestra cualquier reconstrucción de la dimensión pragmática del lenguaje científico, que no sea meramente empírica, es preciso presuponer un vínculo entre los sujetos que lo utilizan, desde el que es posible acordar el uso de los métodos y los términos, comprender el sentido de las proposiciones, la razón de las normas, la constitución de las identidades, la plausibilidad de las interpretaciones, la posible integración del saber. No hay diálogo sobre los objetos, que pueda pretender sentido y validez, si no es desde el reconocimiento intersubjetivo.

Pero cuidar la relación entre los sujetos exige desarrollar un saber hermenéutico, un saber de comprensión e interpretación de esa materia inteligible que es el sentido, que es propio de las ciencias histórico-hermenéuticas. En algún tiempo Apel y Habermas diseñaron aquella teoría de los intereses del conocimiento, según la cual, una teoría crítica trata de desentrañar los intereses que orientan a los distintos saberes, y descubre al menos tres: el interés por dominar y controlar, propio de las ciencias empírico-analíticas, el interés por comprender e interpretar, propio de las ciencias histórico- 
hermenéuticas, y el interés en la emancipación, propio de la crítica de la ideología. Las humanidades tienen por tarea descubrir los intereses que guían el conocimiento, y adentrarse en el terreno de la comprensión, la interpretación y la emancipación, como aquel que les es más propio. Sin duda podrá decirse que las ciencias empírico-analíticas pretenden también la emancipación de los seres humanos y de la naturaleza, pero éste no es un enunciado que pueda comprobarse empíricamente, sino una reconstrucción histórico-hermenéutica, propia de las ciencias humanas (Apel 1985, 28 y 29; Habermas 1984).

Como bien dice Apel, los datos de las ciencias hermenéuticas no son los de una experiencia observacional, sino comunicativa, no solo en el caso de los textos como producto de acciones comunicativas, sino también de las acciones en general, en el sentido de que son concebidos para expresar intenciones cuyo significado puede ser explicado en principio por los propios agentes (Apel 1979).

Aunque Ortega obvia la expresión "Geisteswissenschaften", por entender que existen formas muy diversas de interpretar el término "espíritu", no es menos cierto que las humanidades tienen por objeto la comprensión e interpretación de ese vínculo entre sujetos, de esa posibilidad de comunicación entre ellos, que les capacita para saberse a sí mismos, apropiarse de sí mismos y dirigir la marcha de la historia desde lo que entiendan por progreso. Ése era, a fin de cuentas, el sentido del espíritu hegeliano, la relación entre sujetos, que es más que la suma de ellos, porque abre el camino de la libertad.

En segundo lugar, las humanidades propician la autocomprensión, la construcción de la propia identidad, porque el reconocimiento intersubjetivo lo es de sujetos que se saben diferentes, dotados de distintas identidades, de las que se tienen que apropiar para hacer sus vidas personales.

En tercer lugar, las humanidades propician la formación, el cultivo de la humanidad. En este punto propondrá Herder el ideal de una formación del hombre, que es el elemento en que viven las ciencias del espíritu en el siglo XIX. Los conceptos básicos serán formación, sentido común, capacidad de juicio y gusto, que abre la base de la comunicabilidad.

También permitirán, y ésta será una cuarta ganancia, reflexionar sobre la racionalidad misma y sus formas, porque aunque haya distintos usos de la razón, la razón humana es la misma y es necesario apreciar la unidad del saber. Una de esas formas consistirá en ese uso público de la razón, del que habló Kant en primera instancia y que hoy en día es el núcleo de la teoría deliberativa de la democracia, sea en su versión habermasiana o en la rawlsiana 
(Cortina 2011b). Es el ejercicio de este uso público de la razón el que hace posible construir ciudadanía.

De todo ello podemos inferir que carece de sentido afirmar que es escasa la incidencia de las humanidades en el PIB de un país, que su contribución a la economía nacional es mínima. Precisamente los países más desarrollados trabajan por lograr una excelente formación de los recursos humanos tanto desde el punto de vista del saber científico-técnico como desde los valores de una ciudadanía activa, porque solo la conjunción de ambos puede garantizar en mayor medida el crecimiento, incluso jugando con las reglas del juego de los actuales modelos de crecimiento. No es extraño que en algunas publicaciones universitarias de la Conferencia de Rectores de España, referidas a las innovaciones universitarias, se dedique un apartado a las tecnológicas y otro a las humanísticas, porque ambas suponen un progreso en el conocimiento (RedOTRI Universidades-CRUE, 2011).

Es en esta articulación de las innovaciones en ciencias, técnicas y humanidades en la que nos jugamos el futuro del bien humano.

\section{BIBLIOGRAFÍA}

Apel, Karl-Otto. La transformación de la filosofia. Madrid: Taurus, 1985.

Die Erklären:Verstehen-Kontroverse in transzendental-pragmatischer Sicht.

Frankfurt: Suhrkamp, 1979.

Conill, Jesús. Ética hermenéutica, Madrid: Tecnos, 2006.

Cortina, Adela. Neuroética y Neuropolítica, Madrid: Tecnos, 2011a.

“Ciudadanía democrática: ética, política y religión". Isegoría nº 44 (2011b): 13-55. (ed.). Guía Comares de Neurofilosofía Práctica. Granada: Comares, 2012.

Cortina Adela/Domingo García-Marzá (eds.). Razón pública y éticas aplicadas, Madrid: Tecnos, 2003.

Gadamer, Hans-Georg. Verdad y método. Salamanca: Sígueme, 1977.

Habermas, Jürgen. "Conocimiento e interés". Ciencia y técnica como "ideología” Madrid: Tecnos, 1984. 159-181.

Kagan, Jerome. The Three Cultures: Natural Sciences, Social Sciences and the Humanities in the 21st. Century. Cambridge: Cambridge University Press, 2009.

Ortega y Gasset, José. "Proyecto del Instituto de Humanidades". Obras Completas. Madrid: Revista de Occidente, vol. 7, 1961. 11-24.

(1962): "Boletín número 1 del Instituto de Humanidades". Obras Completas, Madrid: Revista de Occidente, vol. 9, 1962. 439-446. 
Snow, Charles Percy. "La Conferencia Rede, 1959". Las dos culturas y un segundo enfoque. Versión ampliada de "Las dos culturas y la revolución cientifica", Madrid: Alianza, 1977a. 9-61.

"Las dos culturas: un segundo enfoque". Las dos culturas y un segundo enfoque. Versión ampliada de "Las dos culturas y la revolución científica". Madrid: Alianza, 1977b. 63-116.

RedOTRI Universidades-CRUE, 2011, Madrid. 